\title{
A Criterion Characterizing the Orientation of a Vectorcardiogram in Space
}

\author{
H. C. Burger, D.Sc., and J. P. Vaane, M.S., Utrecht, Netherlands
}

In describing and discussing the vectorcardiogram (VCG) there are numerous aspects which merit mention. As it is impossible to consider them all, a selective restriction is mandatory. Among them the concept of the ventricular gradient, which has the direction of the mean heart vector, is now well known. In the case of an elongated loop it nearly coincides with its long axis. In many cases this vague definition has to be replaced by a more exact one, in a way that will not be explained here.

Another criterion of a VCG, often used by different investigators, is the sense of rotation in its projections. The majority of QRS loops of normal vectorcardiograms have a clockwise frontal projection and a counterclockwise horizontal one. If a projection of a VCG has a more or less circular shape, it is easy to determine its sense of rotation. However, if it is very narrow, it often has a figureof-eight shape and an ambiguous direction of rotation. But in space it may be a widely opened loop, which by its position in space is projected as a narrow one, or even as a single line. This is the case if the loop lies in a plane perpendicular to the plane of projection. The position of the plane of the loop in space has been discussed by several investigators. ${ }^{1-3}$ In a recent article, W. R. Milnor ${ }^{4}$ gave a criterion characterizing the position of the QRS plane in space. Using similar reasoning, we have developed a method which is based on the following:

Two projections of a loop, together with their sense of rotation, determine the loop in space with its sense of rotation. The position of the plane of the loop in space, as well as its sense of rotation, can be indicated by one single vector; for example, when the loop lies in a plane, it is a vector perpendicular to this plane and drawn at the side, in which the rotation seen is counterclockwise. Its length indicates the area of the loop and must, therefore, be chosen proportional to it. Its origin may be taken arbitrarily. This vector we will call the polar vector. It may be well to note that this is not the heart vector.

In general, however, the loop does not lie in a plane. Then the definition of the above-mentioned vector can be generalized in such a way that its rectangular components are proportional to the areas of the projections of the loop on the three coordinate planes, an assertion which we shall prove.

From the Department of Medical Physics, Physical Laboratory of the University of Utrecht, Utrecht, Netherlands.

Received for publication March 1, 1958. 
Let $\mathrm{C}$ (Fig. $1, A$ ) be an abritrary closed curve in space. $\mathrm{C}^{\prime}$ is a curved surface circumscribed by $\mathrm{C}$. The three mutually perpendicular axes $\mathrm{F}, \mathrm{H}$, and $\mathrm{S}$ are drawn so that the $\mathrm{H}$ axis is the head-to-foot direction of the patient, the $\mathrm{F}$ axis is perpendicular to the frontal plane of the patient, and $S$ is the sagittal axis.

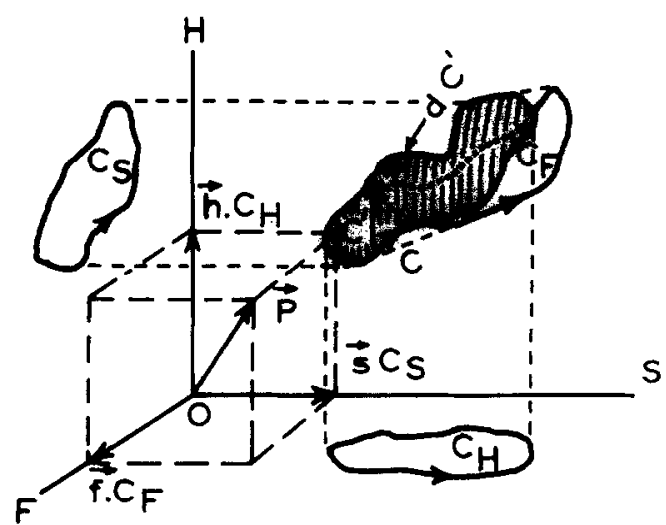

A.

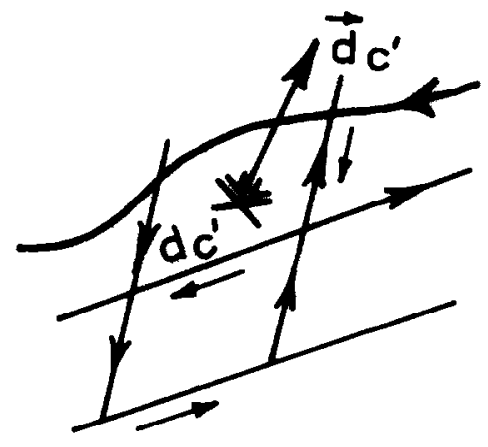

$B$.

Fig. 1. $-A$, The polar vector in relation to an arbitrary QRS loop and its projections. $B$, An infinitesimal surface, $d C^{\prime}$, part of the surface $C^{\prime}$ through the QRS loop, and the accessory infinitesimal polar vector $\overrightarrow{\mathrm{d}}^{\prime}$.

We call the area of the projections of $\mathrm{C}$ on the FS, HS, and $\mathrm{FH}$ planes, respectively, $C_{H}, C_{F}$, and $C_{S} ; \vec{h}, \vec{f}$, and $\vec{s}$ are unit vectors along the positive $H, F$, and $\mathrm{S}$ axes. Now we define as the polar vector, $\overrightarrow{\mathrm{P}}$ of $\mathrm{C}$, the vector

$$
\vec{P}=\vec{h} \cdot C_{\mathrm{H}}+\vec{f} \cdot C_{\mathrm{F}}+\vec{s} \cdot C_{\mathrm{S}}
$$

This vector is by definition independent of the shape of $C^{\prime}$, and perfectly fixed by $\mathrm{C}$ because the projections $\mathrm{C}_{\mathrm{H}}, \mathrm{C}_{\mathrm{F}}$, and $\mathrm{C}_{\mathrm{S}}$ are so.

Next, we define still another vector, $\vec{P}^{\prime}$. Herein we divide $C^{\prime}$ into infinitesimal surfaces, $\mathrm{dC}^{\prime}$, the contours of which run in the same sense as the QRS-loop $\mathrm{C}($ Fig. $1, B)$. To every surface $\mathrm{dC}^{\prime}$ we define an infinitesimal vector, $\overrightarrow{\mathrm{dC}}^{\prime}$, with a direction and length corresponding in the same way to the sense of rotation and area of $\mathrm{dC}^{\prime}$, if the loop lies in a plane, as the polar vector $\overrightarrow{\mathrm{P}}$ would correspond to $\mathrm{C}$. By integration over the surface $\mathrm{C}^{\prime}$ we define:

and $\overrightarrow{\mathrm{P}}^{\prime}$ is fixed by the shape of $\mathrm{C}^{\prime}$.

$$
\vec{P}^{\prime}=\int_{C^{\prime}} \overrightarrow{d C}^{\prime}
$$

Now, if we prove that $\overrightarrow{\mathrm{P}}=\overrightarrow{\mathrm{P}}^{\prime}$ for every arbitrary surface circumscribed by $\mathrm{C}$, the truth of our theorem, "the rectangular components of the polar vector are proportional to the areas of the projections of the QRS loop on the coordinate planes," is established.

Let $\mathrm{C}$ again be a closed curve in space, and $\mathrm{C}^{\prime}$ a curved surface circumscribed by $\mathrm{C}$ (Fig. 2). We require that $\mathrm{C}$ and $\mathrm{C}^{\prime}$ are a curve and a surface, which can be differentiated and integrated, and which are, therefore, continuous. Once more we divide $\mathrm{C}^{\prime}$ into infinitesimally small surfaces, $\mathrm{dC}^{\prime}$, with the corresponding vectors $\overrightarrow{\mathrm{dC}}^{\prime}$ having a length proportional to the area of $\mathrm{dC}^{\prime}$. Looking at 
the projection $C_{F}$ of $C^{\prime}$ it may be readily seen that for every surface $\mathrm{dC}^{\prime}$ lying at the "front" of $\mathrm{C}^{\prime}$ a surface $\mathrm{dC}^{\prime}{ }_{1}$ corresponds at the "back" of $\mathrm{C}^{\prime}$, with exactly the same projection on the $\mathrm{HS}$ plane, except for the $\mathrm{dC}_{1}^{\prime}$ 's with their projection lying at or within $\mathrm{C}_{\mathrm{F}}$. Because the corresponding $\mathrm{dC}^{\prime \prime} \mathrm{s}$ and $\mathrm{dC}_{1}{ }_{1}$ 's have opposite signs, $C_{F}$ fixes the projection of $C^{\prime}$ on the HS plane. If $C^{\prime}$ is so shaped that the projection of a part of its "front" falls within $\mathrm{C}_{F}$, there must be another part, its "back," with the same projection and opposite sign, because of the fact that the curve and the surface are continuous. Therefore, in that case, $\mathrm{C}_{F}$ will also be "the" projection of $\mathrm{C}^{\prime}$ on the HS plane.

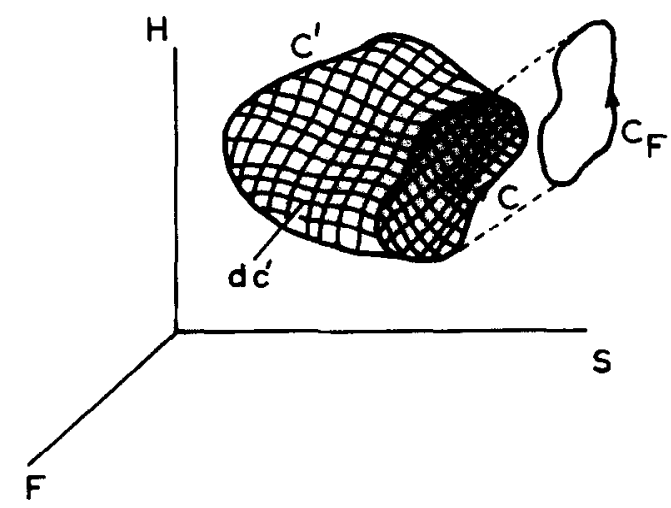

Fig. 2.-An arbitrary surface circumscribed by a QRS loop.

In the same way we prove that $\mathrm{C}_{\mathrm{S}}$ and $\mathrm{C}_{\mathrm{H}}$ are "the" projections on the $\mathrm{FH}$ and $\mathrm{SF}$ planes, respectively. Now let $\mathrm{dC}_{\mathrm{S}}^{\prime}, \mathrm{dC}_{\mathrm{F}}^{\prime}$, and $\mathrm{dC}_{\mathrm{H}}^{\prime}$ be the projections of $\mathrm{dC}^{\prime}$ on the $\mathrm{FH}, \mathrm{SH}$, and $\mathrm{SF}$ planes, respectively; then the vector $\overrightarrow{\mathrm{dC}}^{\prime}$ is fixed by the equation:

$$
\overrightarrow{d C}^{\prime}=\vec{h} \cdot d C_{\mathrm{H}}^{\prime}+\vec{f} \cdot d C_{\mathrm{F}}^{\prime}+\vec{s} \cdot d C_{\mathrm{S}}^{\prime}
$$

The polar vector $\overrightarrow{\mathrm{P}}^{\prime}$ of $\mathrm{C}$ is the integral of the $\overrightarrow{\mathrm{dC}}$ "s over $\mathrm{C}^{\prime}$ :

$$
\overrightarrow{P^{\prime}}=\underset{C^{\prime}}{\int_{d C}} \overrightarrow{d C} \cdot \underset{C_{\mathrm{H}}^{\prime}}{\int_{\mathrm{H}}} d C_{\mathrm{H}}^{\prime}+\vec{f} \cdot \underset{C_{\mathrm{F}}^{\prime}}{\int_{\mathrm{F}}} d C_{\mathrm{F}}^{\prime}+\vec{s} \cdot \underset{C_{\mathrm{S}}^{\prime}}{\int_{C}} d C_{\mathrm{S}}^{\prime}
$$

The three integrals are apparently the areas of $C_{H}, C_{F}$, and $C_{S}$, respectively.

Thus the following equation holds:

$$
\overrightarrow{P^{\prime}}=\vec{h} \cdot C_{\mathrm{H}}+\vec{f} \cdot C_{\mathrm{F}}+\vec{s} \cdot C_{\mathrm{S}}
$$

and the right side is the vector, with the components being the projections of the curve $\mathrm{C}$ on the coordinate planes. Therefore, we have proved the aforementioned theorem: "The rectangular components of the polar vector are proportional to the areas of the projections of the QRS loop on the coordinate planes."

The evaluation of the vector $\vec{P}$ is easy if the three rectangular projections of the QRS loop of a vectorcardiogram are recorded. Their areas give the three components of the previously mentioned polar vector. 
In order to obtain the three rectangular projections of the QRS loop, we connected a patient to a vectorcardiograph. The essential property of this apparatus is that the leads (the voltages measured between the electrodes when connected to the patient) are amplified, attenuated, and combined by sets of

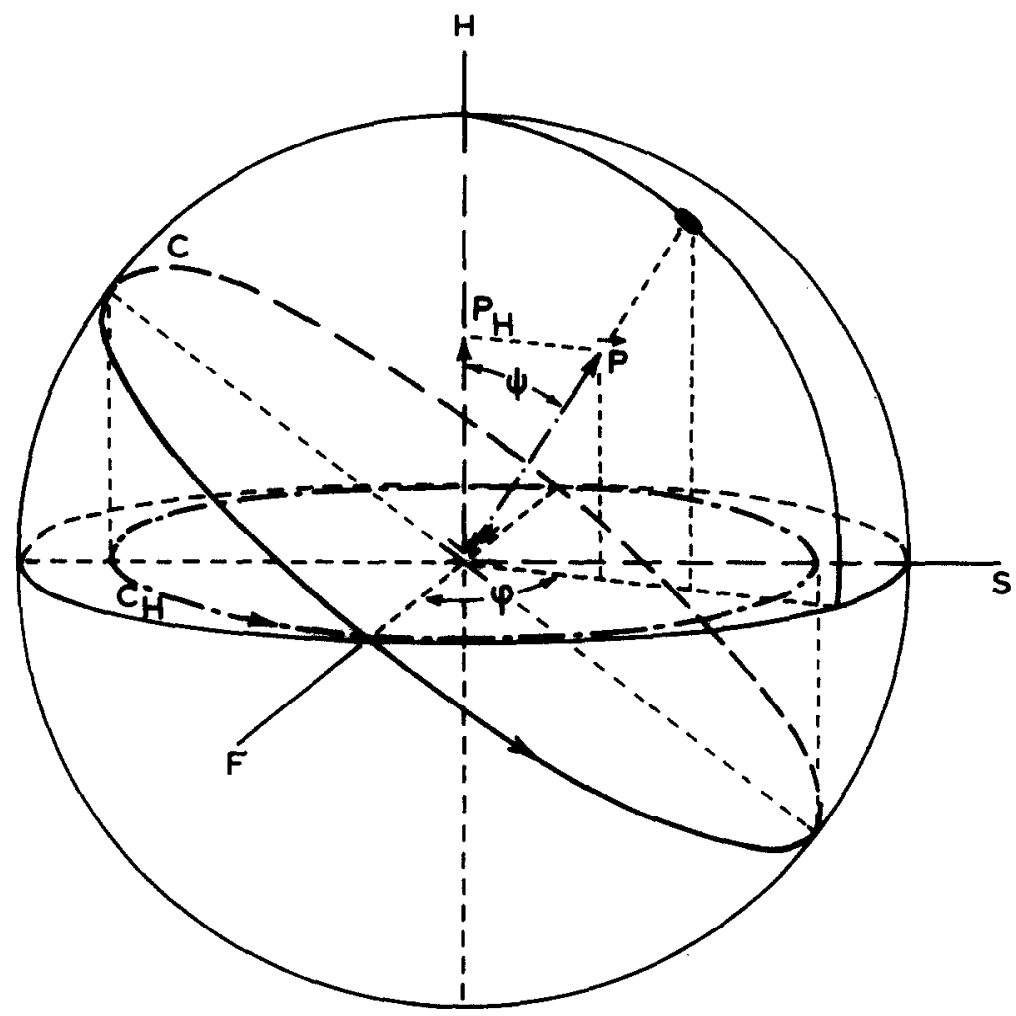

Fig. 3. -The method used to determine the coordinates $\varphi$ and $\Psi$ of the polar vector from the projections of the QRS loop.

resistances, so that finally the three perpendicular projections of the vectorcardiogram appear successively on the screen of a cathode-ray tube by selecting the appropriate position of a switch. The lead system we used is the $B_{1}$ system, which we published years ago. In this system the electrodes are fixed on the left and the right upper arm, on the sternum at the height of the axilla, and on the right leg. In Fig. 3 a QRS-loop $C$ is drawn, situated arbitrarily in space. In the same picture the three mutually perpendicular axes $\mathrm{F}, \mathrm{H}$, and $\mathrm{S}$ are drawn. Only one projection of the loop $\mathrm{C}$, the horizontal one, $\mathrm{C}_{\mathrm{H}}$, is drawn, in order to keep the picture as clear as possible.

Now let $\overrightarrow{\mathrm{P}}$ be the polar vector, its length being proportional to the area of C. Because of the vector character of $\vec{P}, P_{H}$ being its component along the $H$ axis, the length of $\mathrm{P}_{\mathrm{H}}$ is proportional to the area of $\mathrm{C}_{\mathrm{H}}$. In a similar way the length of the other two components of $\overrightarrow{\mathrm{P}}, \mathrm{P}_{\mathrm{F}}$ and $\mathrm{P}_{\mathrm{S}}$, indicate the areas of the projections $C_{F}$ and $C_{S}$, respectively. 
The projections $\mathrm{C}_{H}, \mathrm{C}_{\mathrm{F}}$, and $\mathrm{C}_{\mathrm{S}}$ now appear at will on the screen of the cathode-ray tube. Thus, by making a picture of the screen we obtain the length of the components $P_{H}, P_{F}$, and $P_{S}$ from which the angles $\varphi$ and $\Psi$, determining the direction of $\overrightarrow{\mathrm{P}}$, can be computed (Fig. 3 ).

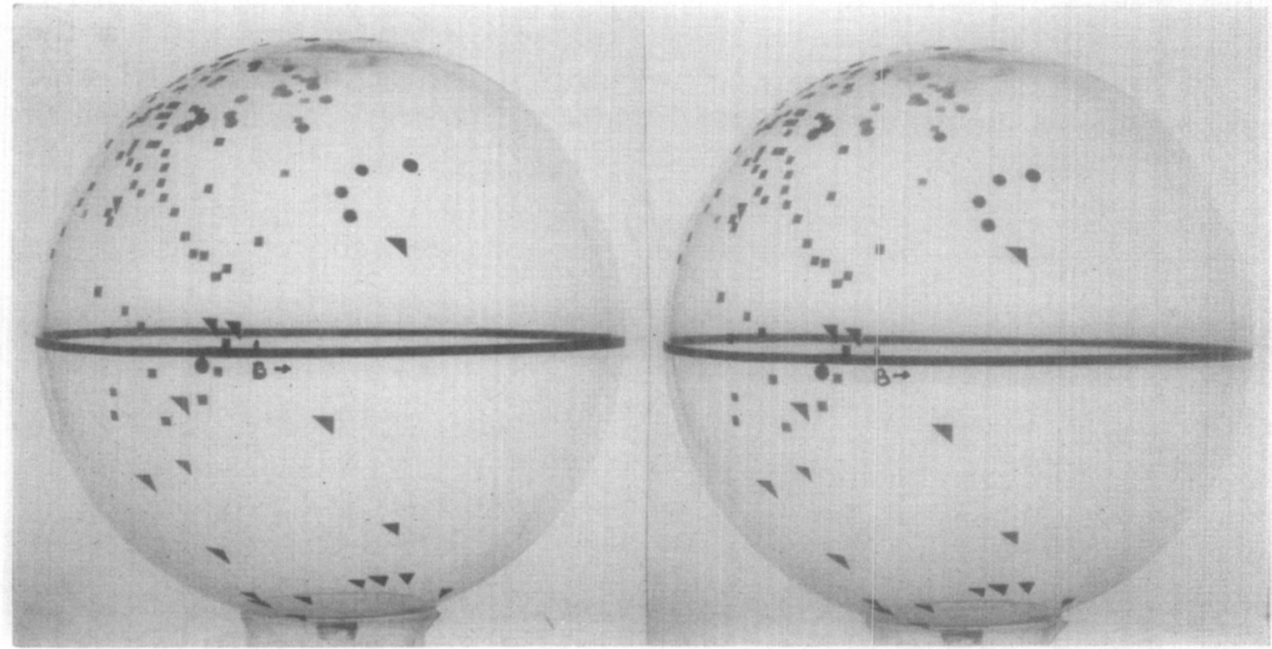

Fig. 4A, -Stereoscopic picture of the globe. $\quad=$ Normal cases $(100) . \quad \bullet=$ Left ventricular hypertrophy (23). $\quad=$ Right ventricular hypertrophy (19). 'The letter B indicates the back $\left(180^{\circ}\right)$ of the patient.

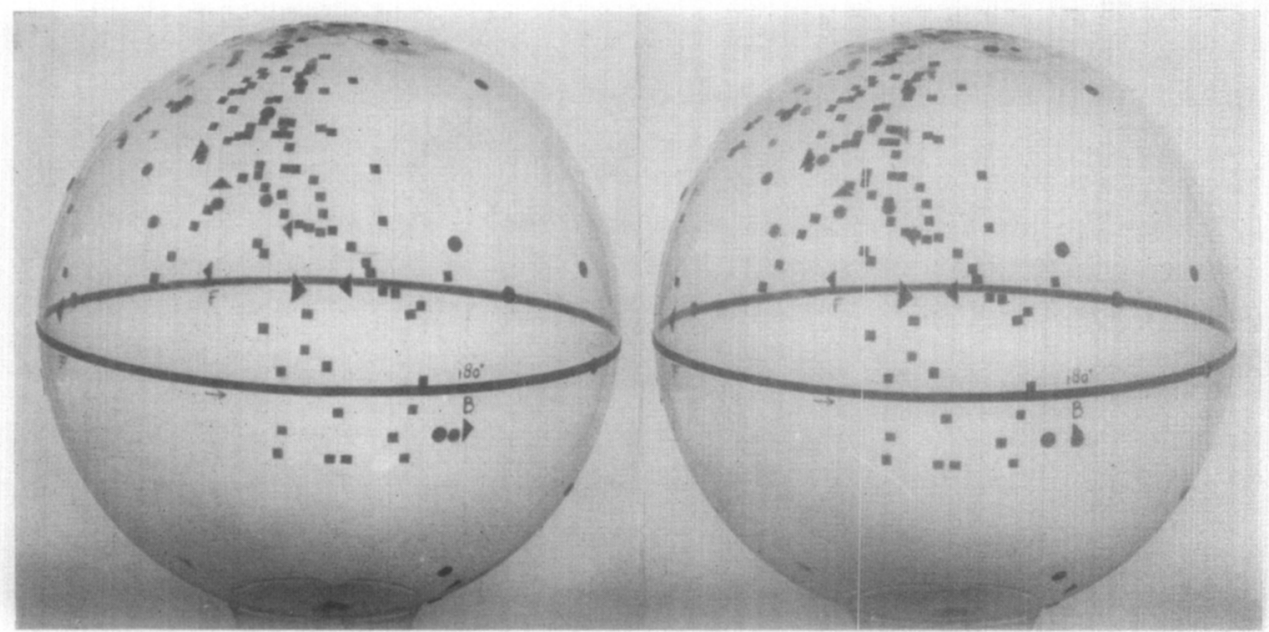

Fig. 4B.-Stereoscopic picture of the globe. $=$ Normal cases (104). $\quad=$ Left bundle branch block (7). = Right bundle branch block (13). - = Othor disturbances in conduction (5). Infarcts (22). The letters $B$ and $F$ indicate, respectively, the back $\left(180^{\circ}\right)$ and the front $\left(0^{\circ}\right)$ of the patient. 
Since the polar vector is drawn at that side of the loop $\mathrm{C}$ from which its sense of rotation is seen counterclockwise, the signs of the components of $\vec{P}$ are fixed by the direction of the polar vector and are obtained in the same way as the direction of $\overrightarrow{\mathrm{P}}$ is obtained from the sense of rotation of $C$.

By the method described above we took vectorcardiograms from some 200 subjects, some with pathologic heart conditions, the rest normal, and calculated the direction $(\varphi, \Psi$ in Fig. 3$)$ of the polar vector. We did not take into consideration the length of $\overrightarrow{\mathrm{P}}$, but let them all have the same arbitrary length, so that they could be represented by points on a sphere, analogous to the method which Brinberg ${ }^{7}$ used for determining the situation of the gradient. We divided the cases into normal, left ventricular hypertrophy, right ventricular hypertrophy, left and right bundle branch block, other disturbances in conduction, and infarct. The cases had been diagnosed by cardiologists who employed the usual clinical means, including electrocardiography.

In order to obtain a clear picture of the directional distribution of the polar vectors, we used two glass globes. On one of these $(A)$ we put the normal cases and those of left and right ventricular hypertrophy; on the other globe $(B)$ we put the normal cases, and those of left and right bundle branch block, other disturbances in conduction, and infarcts. Figs. $4 A$ and $4 B$ give a stereoscopic picture of these two globes. They represent the data just as well as a list of values of the angles $\varphi$ and $\Psi$, which we will not reproduce here.

\section{CONCLUSION}

Seventy-five per cent of the normal cases lie in the octant given by $90^{\circ}<\varphi<180^{\circ}, \Psi<90^{\circ}$.

Sixty-three per cent of the right ventricular hypertrophies lie below the equator, having $\varphi$ near $180^{\circ}$ (back); the left ventricular hypertrophies lie on the upper half of the globe with $\varphi$ near $0^{\circ}$ (front).

Of the other cases 80 per cent lie outside the "normal" area, 50 per cent of them lying in or near the area of left hypertrophy.

The value of the method in diagnosing a case may be described as follows: Having computed the angles $\varphi$ and $\Psi$ representing the direction of the polarvector for a certain case, the probability of the patient having a particular abnormality (or being normal) is proportional to the density of points in the cloud on the sphere, which represents this abnormality at the point found for the particular patient.

As in other diagnostic methods, the final diagnosis is better established only after more facets of the disease are investigated. The position of the QRS loop in space is meant to be only one of them.

\section{SUMMARY}

The orientation of the plane of the QRS loop of a vectorcardiogram can be described by its polar vector. In a number of cases (normal as well as abnormal) the direction of this vector has been determined and displayed on a globe. The distribution of points on this globe may be of help in diagnosing the abnormality from the vectorcardiogram. 
We wish to express our thanks to J. B. van Milaan, Ph.D., and A. G. W. van Brummelen, M.S., for their help with the development of the method, and to H. Schneider, B.S., for collecting a great part of the data.

\section{REFERENCES}

1. Abildskov, J. A.: Circulation 12:286, 1955.

2. Burch, G. E., Abildskov, J. A., and Cronvich, J. A.: Circulation 7:558, 1953.

3. Grant, R. P., and Estes, E. H.: Spatial Vector Electrocardiography, New York, 1951, Blakiston.

4. Milnor, W. R.: Circulation 16:95, 1957.

5. Seiden, G. E.: Circulation 14:999, 1956.

6. Burger, H. C., van Milaan, J. B., and den Boer, W.: Brit. Heart J. 14:401, 1952.

7. Brinberg, L.: J. Mt. Sinai Hosp. 23:751, 1956. 